\title{
Differential Protein Profiling of
} Cerebrospinal Fluid in Piglets with Severe Meningoencephalitis Caused by Streptococcus suis Type 2 Compared to Controls

\section{OPEN ACCESS}

Edited by:

Margaret E. Bauer, School of Medicine, Indiana University Bloomington, United States

Reviewed by:

Guoqiang Zhu,

Yangzhou University, China Karl Bechter,

University of UIm, Germany

${ }^{*}$ Correspondence: Liancheng Lel

leilc@jlu.edu.cn leiliancheng@163.com

${ }^{\dagger}$ Co-first author.

Received: 26 September 2017 Accepted: 25 January 2018

Published: 09 February 2018

Citation: Liu H, Jia L, Guo W, Sun Y, Zhu R, Li S, Qu G, Jiang H, Wang J, Gu J, Sun $C$, Feng $X$, Han $W$ and Lei $L$ (2018) Differential Protein Profiling of

Cerebrospinal Fluid in Piglets with Severe Meningoencephalitis Caused by Streptococcus suis Type 2 Compared to Controls.

Front. Cell. Infect. Microbiol. 8:35 doi: 10.3389/fcimb.2018.00035

\begin{abstract}
Hongtao Liu ${ }^{1}$, Li Jia ${ }^{1 \dagger}$, Wenfei Guo ${ }^{1}$, Yingying Sun ${ }^{1}$, Rining Zhu ${ }^{1}$, Shuguang $\mathrm{Li}^{2}$, Guanggang $\mathrm{Qu}^{2}$, Hexiang Jiang ${ }^{1}$, Junjie Wang ${ }^{1}$, Jingmin $\mathrm{Gu}^{1}$, Changjiang Sun ${ }^{1}$, Xin Feng ${ }^{1}$, Wenyu Han ${ }^{1}$ and Liancheng Lei ${ }^{1 *}$

${ }^{1}$ College of Veterinary Medicine, Jilin University, Changchun, China, ${ }^{2}$ Shandong Binzhou Animal Science and Veterinary Medicine Academy, Binzhou, China
\end{abstract}

Streptococcus suis serotype 2 (SS2) is a zoonotic pathogen that can cause meningitis both in pigs and in human beings. However, the pathogenesis of central nervous system (CNS) infection caused by SS2 have not yet been elucidated. To find the key molecules in cerebrospinal fluid (CSF) needed for the pathogenesis, a SS2 meningoencephalitic pig model and a SS2 non-meningoencephalitic pig model were established in this study. CSF was collected from infected piglets, and protein profiling was performed with label-free proteomics technology. A total of 813 differential proteins, including 52 up-regulated proteins and 761 down-regulated proteins, were found in the CSF of meningoencephalitic pigs compared with both non-meningoencephalitic pigs and healthy pigs. These 813 differential proteins were clustered into three main categories, namely, cellular component, biological process, and molecular function by gene ontology (GO) analysis. The most enriched subclasses of differential proteins in each category were exosome (44.3\%), energy pathway (25.0\%) and catalytic activity (11.3\%), respectively. The most enriched subclasses of upregulated proteins were extracellular (62.1\%), protein metabolism (34.5\%) and cysteine-type peptidase activity (6.9\%), and of downregulated proteins were exosomes (45.0\%), energy pathway (24.0\%) and catalytic activity (9.4\%). Then, the differential proteins were further investigated by using the KEGG database and were found to participate in $16 \mathrm{KEGGs.} \mathrm{The} \mathrm{most} \mathrm{enriched} \mathrm{KEGG} \mathrm{was} \mathrm{citrate}$ cycle (56.6\%), and some of these differential proteins are associated with brain diseases such as Huntington's disease (18.6\%), Parkinson's disease (23.8\%) and Alzheimer's disease (17.6\%). Sixteen of the 813 differential proteins, chosen randomly as examples, were further confirmed by enzyme-linked immunosorbent assay (ELISA) to support the proteomic data. To our knowledge, this is the first study to analyze the differential protein profiling of CSF between SS2 meningoencephalitic piglets and non-meningoencephalitic piglets by employing proteomic technology. The discovery and bioinformatics analysis of these differential proteins provides reference data not only for research on pathogenesis of SS2 CNS infection but also for diagnosis and drug therapy research.

\footnotetext{
Keywords: Streptococcus suis, meningoencephalitis, blood-CSF barrier, cerebrospinal fluid, proteomics
} 


\section{INTRODUCTION}

Streptococcus suis type 2 is a zoonotic pathogen that can cause meningitis, bacteremia, septicemia, endocarditis, and arthritis in pigs and humans. Meningitis is the most serious symptom of $S$. suis 2 infection and often shows clinical symptoms including vertigo, ataxia, headaches, neck stiffness, fevers, nausea, deafness and so on (Madsen et al., 2002; Mai et al., 2008; van Samkar et al., 2015; Dejace et al., 2017; Sena et al., 2017), and histopathological features including the presence of fibrin, edema and cell infiltration in the meninges and choroid plexus. As a result, this pathogen poses a great threat not only to the swine industry but also to human health.

Obtaining effective and controllable drugs that can pass through the blood-CNS barrier to treat neurological diseases remains a challenge for modern medicine (Coureuil et al., 2017). Understanding the pathogenesis of SS2 meningitis is critical for identifying candidate targets for the diagnosis and therapy of SS2 meningitis. SS2 can cause meningitis due to the interaction between SS2 virulence factors and host molecules. Therefore, not only do the virulence factors from SS2 play an important role in the process of SS2 causing meningitis, but host molecules are also very important for this process.

The blood-CNS barrier, including BCB (blood-CSF barrier) and $\mathrm{BBB}$ (blood-brain barrier), is important for maintaining CNS homeostasis. The prerequisite of SS2 causing meningitis is SS2 penetration into the blood-CNS barrier. The blood-CSF barrier is composed of three interfaces: the choroid plexuses (CPs), the pial microvessels and the arachnoid barrier cell layer (Brochner et al., 2015). The CSF is secreted by the CPs, which are villous structures attached to the ventricular ependyma (Strazielle and Ghersi-Egea, 2000). Many studies have reported that some molecules in CSF could be used as potential biomarkers of some brain diseases and bio-targets of therapeutic drugs and pathogenesis research. For example, the porcine cathelicidin PR39 was found to be significantly increased in CSF of piglets with meningitis and could inhibit neutrophil DNA degradation by bacterial nucleases (de Buhr et al., 2017). Insulin concentrations often found to be decreased in CSF of Alzheimer's disease (AD) patients and could providing insights for $\mathrm{AD}$ pathogenesis research (Craft et al., 1998). Comprehensive proteome profiles of different grades of gliomas using CSF could providing insights into disease pathobiology and differentially abundant CSF proteins may serve as potential disease monitoring and prognostic markers for gliomas (Gahoi et al., 2017). The findings of these studies suggested that the discovery of the differential protein in CSF could provide important candidate biological targets for the research of diagnosis, pathogenesis and drug therapy of brain diseases.

Label-free quantification is a method in mass spectrometry (MS). Unlike other methods for protein quantification, labelfree quantification does not use a stable isotope to chemically bind to and thus label the protein. This method was widely used to determine the relative amount of proteins in two or more biological samples (Bantscheff et al., 2007; Asara et al., 2008). UniProt is a freely accessible database of protein sequence and functional information, which was built by UniProt consortium that comprises the European Bioinformatics Institute (EBI), the Swiss Institute of Bioinformatics (SIB), and the Protein Information Resource (PIR), many entries being derived from genome sequencing projects. It contains a large amount of information about the biological function of proteins derived from the research literature (O'Donovan et al., 2002; Boeckmann et al., 2003; Wu et al., 2003).

There are no previous reports about the protein profiling of cerebrospinal fluid in meningitic pigs. For this purpose, SS2 meningoencephalitic and non-meningoencephalitic pig models were established in this study. CSF from different pig models was collected and analyzed by protein profiling using label-free proteomics technology. The data of proteomic analyses are useful for research of SS2 CNS infection diagnosis, pathogenesis and therapy.

\section{MATERIALS AND METHODS}

\section{Ethics Statement}

Bama miniature pigs used for infection experiments were purchased from Shandong Binzhou Animal Science Veterinary Medicine Academy. All animal experimental procedures were performed in strict accordance with the Regulations for the Administration of Affairs Concerning Experimental Animals approved by the State Council of the People's Republic of China (1988.11.1).

\section{Bacterial Strains and Culture Conditions}

SS2 strains used in this study were isolated from sick pigs. The JZLQ022 strain was isolated from brain tissue of meningitis pig, and the JZLQ001 strain was from lymphonodi mandibulares of arthritis pig. SS2 strains were grown on tryptic soy agar (TSA) plates with 5\% newborn bovine serum (CLARK Co. Ltd., Australia) for $10 \mathrm{~h}$ at $37^{\circ} \mathrm{C}$. Then isolated colonies were transferred into $3 \mathrm{ml}$ of tryptic soy broth (TSB) with 5\% newborn bovine serum and incubated for $8 \mathrm{~h}$ at $37^{\circ} \mathrm{C}$ with agitation. The bacterial pellet was obtained by centrifugation and resuspended in phosphate-buffered saline (PBS) with a continuous 10fold dilution. These dilutions were plated onto TSA plates to accurately determine the CFU per milliliter.

\section{The Establishment of a Porcine Model of SS2 Infection}

Eighteen 26-day-old healthy inbred Bama miniature pigs were randomly divided into three groups $(n=6)$ : meningoencephalitis group $\left(200 \mu \mathrm{L}\right.$ PBS with $5 \times 10^{6}$ CFUs JZLQ022), nonmeningoencephalitis group $\left(200 \mu \mathrm{L}\right.$ PBS with $5 \times 10^{6}$ CFUs JZLQ001) and healthy group (200 $\mu \mathrm{L}$ PBS) by ear intravenous injection. Clinical symptoms including appetite, mental status and body temperature were continuously monitored post infection.

\section{Collection of Samples and Determination of Viable Bacteria in Organs}

Heparinized blood was collected $2 \mathrm{~h}, 4 \mathrm{~h}, 12 \mathrm{~h}, 2 \mathrm{~d}, 3 \mathrm{~d}, 5 \mathrm{~d}$, and $7 \mathrm{~d}$ after infection. The pigs were euthanized with sodium pentobarbital $(400 \mathrm{mg} / \mathrm{mL}$ at a dose of $500 \mu \mathrm{L} / \mathrm{kg})$ by ear 
intravenous injection when pigs were dying. After euthanasia, skin and muscles in the back of neck was cut and foramen magnum was exposed. $10 \mathrm{ml}$ injector was inserted into the foramen magnum keep away from spinal cord to collect the CSF. Brain tissue was collected after opening the cranial cavity. $1 \mathrm{~g}$ of brain tissue was made into homogenate with $500 \mu \mathrm{L}$ PBS and plated onto TSA plates to accurately determine the CFU per gram.

\section{Liquid Chromatography-Mass Spectrometry (LC-MS/MS) Measurements} The CSF samples were added to appropriate doses of lysis buffer (7 M Urea, $2 \mathrm{M}$ Thiourea, 0.1\% CHAPS) and vortex blended followed by incubation for $30 \mathrm{~min} \mathrm{~s}$ at $25^{\circ} \mathrm{C}$. Then, the sample was centrifuged at $15,000 \mathrm{~g}$ for $20 \mathrm{~min}$ at $4^{\circ} \mathrm{C}$. The supernatant was subpackaged into a $1.5 \mathrm{~mL}$ tube. The concentration of total protein in the supernatant was determined by Bradford method (Bradford, 1976). LC-MS/MS was performed at the QLbio Biotechnology Corporation in Beijing using the Thermo Scientific EASY-nLC 1000 System and Thermo Q-Exactive MS system. Raw data were identified by Maxquant software and the Sus scrofa protein database on the Uniprot Website (http://www. uniprot.org/).

\section{Proteomic Data Analysis}

Fold changes in proteins were determined according to labelfree quantitative (LFQ) intensity between the meningitic group and non-meningitic group or healthy group. Proteins with a difference $>1.5$-fold or $<0.66$-fold, $p$-values $<0.05$ were regarded as differentially abundant proteins. Functional analysis of differential proteins was performed based on the gene ontology (GO) annotations (http://www.geneontology.org/), and the proteins were categorized according to their cellular components, molecular functions and biological processes (http://pantherdb. org/geneListAnalysis.do). The differential proteins were further assigned to the Kyoto Encyclopedia of genes and genomes (KEGG) database (http://kobas.cbi.pku.edu.cn/anno_iden.php).

\section{Validation with Enzyme-Linked Immunosorbent Assay (ELISA) Analyses}

Some differential proteins in CSF from differential infected piglets, including 7 up-regulated proteins (CTSL1, CTSZ, SAA, MMP-2, RBP-4, CHGA, and CuZnSOD) and 9 downregulated proteins (Rock, Clathrin, Vinculin, Arp2/3, RhoA, Bid, CytC, PKC, and ERK), were detected by double-antibody sandwich ELISA kits (Purchased from Shanghai Jin Ma Biological Technology co., LTD).

\section{RESULTS}

\section{Establishment of a Pig Model of SS2 Meningoencephalitis}

Decreased appetite and depressed emotion were observed both in the two groups of pigs infected with JZLQ022 and JZLQ001, respectively. Hyperpyrexia was monitored, and the body temperature of some pigs in the two groups was up to $40^{\circ} \mathrm{C}$ at $24 \mathrm{~h}$. Arthritis symptoms, including limp, trouble walking and arthrocele, were observed in some pigs infected with JZLQ001 at 3 d (Supplementary Material Video 1). Trouble walking was also observed in some pigs infected with JZLQ022 at $4 \mathrm{~d}$, and foaming at the mouth and ataxia was observed at $5 \mathrm{~d}$ (Supplementary Material Videos 2, 3). By autopsy, conspicuous macroscopic lesions, including lung purulent lesions, spleen swelling and infarction, and kidney nephremia, were observed both in the JZLQ022 and JZLQ001 groups (Figure 1A). In addition, joint effusion was observed in the JZLQ001 group. In accordance with the macroscopic lesions, brain hyperemia and hemorrhage were observed in the JZLQ022 group, but not in the JZLQ001 group (Figure 1A). Collected blood and brain tissues were used for bacterial counting. The JZLQ022 infection group had higher SS2 CFUs than PBS control group and the JZLQ001 group in blood at $2 \mathrm{~d}, 3 \mathrm{~d}$ (Figure 1B) and in brain tissues (Figure 1C). The pig models of meningoencephalitis and non-meningoencephalitis were successfully established.

\section{Comprehensive Analysis of Proteomic Data}

The CSF of pigs from the three treatment groups was collected and analyzed by label-free proteomics technology at the QLbio Biotechnology Limited Company (Beijing, China). Each sample was analyzed in triplicate. A total of 3456 proteins were authenticated with LC-MS/MS technology and calculated by iBAQ and LFQ algorithms with Maxquant software. The notes of these proteins can be found in Uniprot database. The analysis results show good reproducibility based on the heat-map (Figure 2A) and similarity (the more approximate the value is to 1 , the higher the similarity between the two sets of data will be) (Figure 2B). Most molecules in CSF from the meningitic group present lower detection concentrations (green) compared with the other two groups, and a small number of molecules present high detection concentrations (red) (Figure 2A).

Comparison of the changes in proteins between pairs of groups resulted in some similarities and considerable differences. The Venn diagram shows the distribution of the proteins between each pair of groups. Differentially expressed proteins were detected between each pair of groups. Among these proteins, 255 proteins were differentially distributed for all three comparisons (meningoencephalitis vs. nonmeningoencephalitis, $\mathrm{M} / \mathrm{N}$; meningoencephalitis vs. healthy, $\mathrm{M} / \mathrm{H}$; non-meningoencephalitis vs. healthy, N/H). A total of 447 (255 plus 192) co-differential proteins were found when analyzing $\mathrm{M} / \mathrm{H}$ against $\mathrm{N} / \mathrm{H}$. These 447 proteins may provide hints for SS2 infection related research. A total of 813 (255 plus 558) co-differential proteins were found when analyzing $\mathrm{M} / \mathrm{N}$ against $\mathrm{M} / \mathrm{H}$ (Figure $2 \mathrm{C}$ ). These 813 proteins may provide hints for SS2 meningitis related research and are called 813CDPs in the following.

\section{GO Analysis and Classification of the 813CDPs}

The 813CDPs were clustered into three main categories, namely, biological process, cellular component, and molecular function, by gene ontology (GO) analysis. The top 10 most enriched GO terms were selected in each category. The $10 \mathrm{GO}$ terms in cellular component contained exosomes 

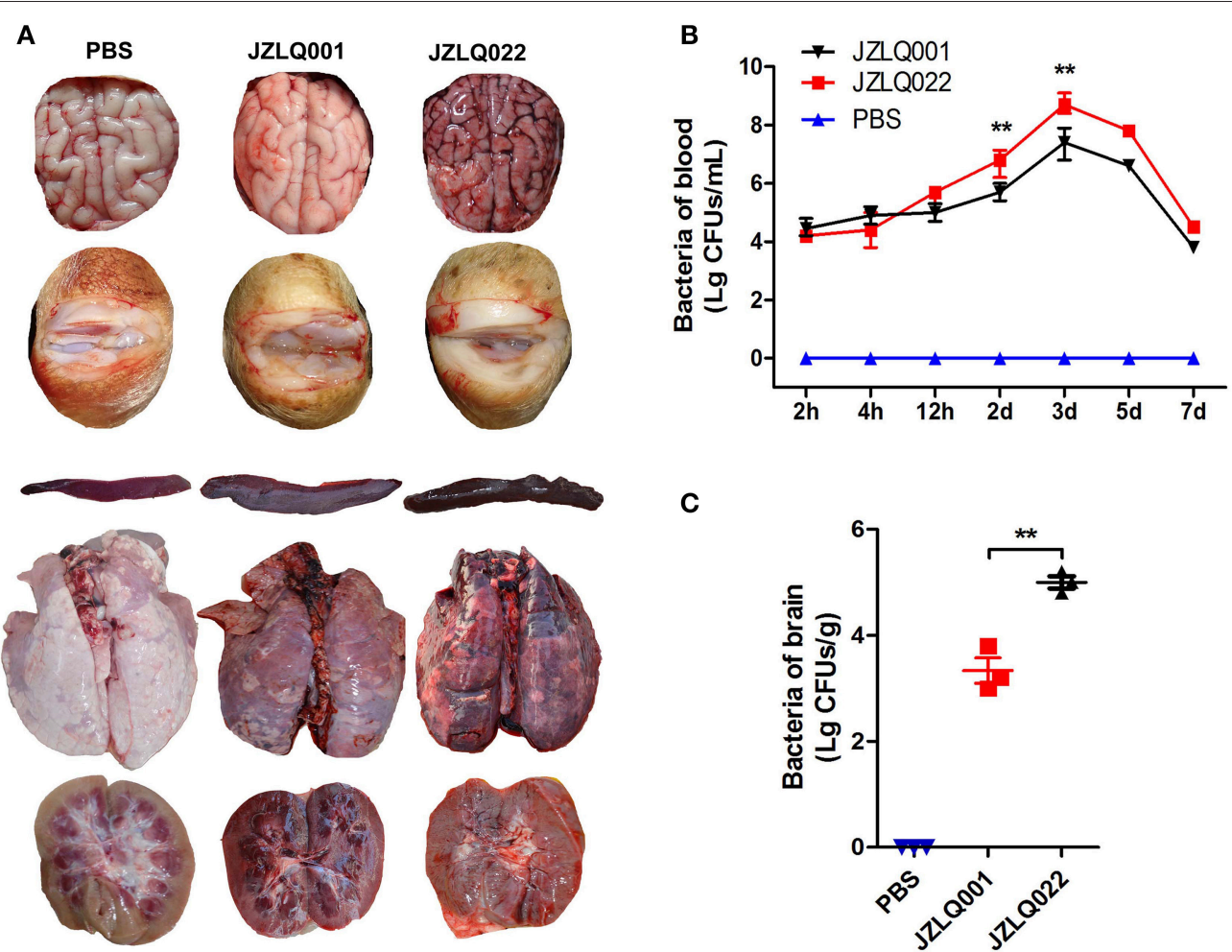

C

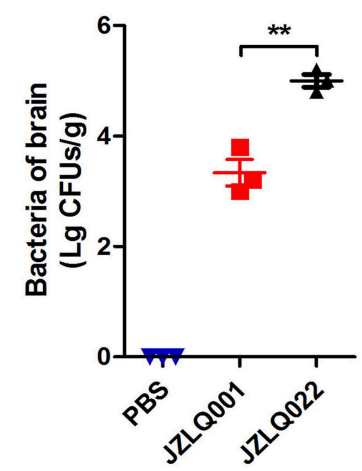

FIGURE 1 | Establishment of SS2 meningoencephalitis and non-meningoencephalitis pig models. (A) Autopsy results of brain and arthrosis; (Control: pig infected with PBS representing the healthy group; JZLQ001: pig infected with the JZLQ001 strain representing the non-meningoencephalitic group; JZLQ022: pig infected with the JZLQ022 strain representing the meningoencephalitic group). (B) Bacteria counts in blood collected at different time points after infection with SS2 ( $n=6$; ${ }^{* *} p$ $<0.01)$. (C) Bacteria counts in brain tissue collected at different time points after infection with SS2 $\left(n=3 ;{ }^{* *} p<0.01\right)$.

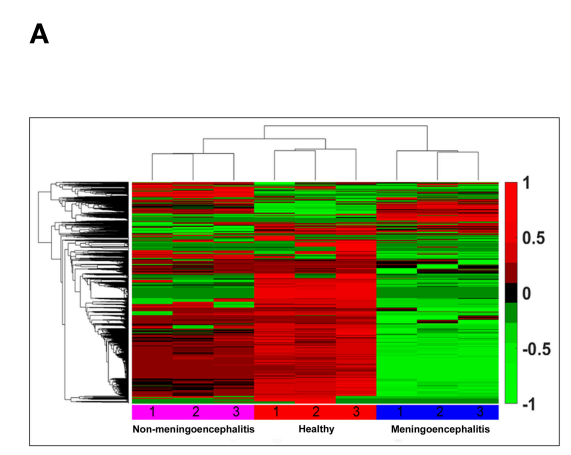

B

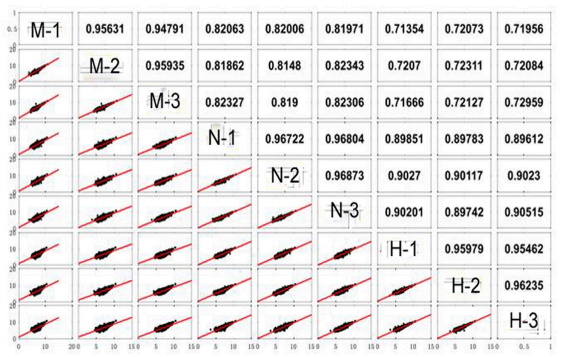

C

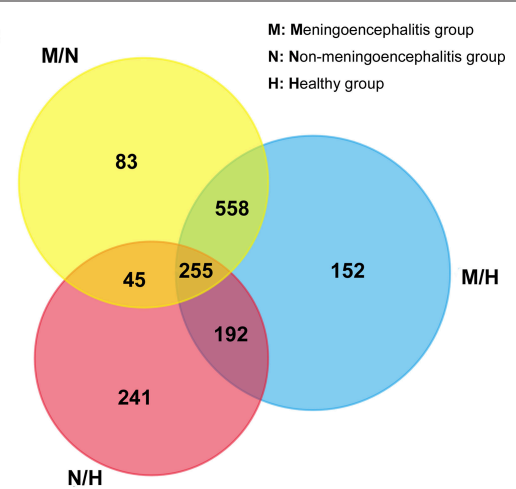

FIGURE 2 | Comprehensive analytic results of proteomic data. (A) The heat-map of proteomics results. (Red, 1: up-regulated proteins; Green, -1 : down-regulated proteins; Black, O: Unchanged proteins). (B) Repetitive analysis of proteomic data. (M, meningoencephalitic group; N, non-meningoencephalitic group; H, Healthy group). (C) Venn diagram of the number of differential proteins among the three treatment groups. $\mathrm{M} / \mathrm{N}$, Differential proteins between the meningoencephalitic group and non-meningoencephalitic group; $\mathrm{M} / \mathrm{H}$, Differential proteins between the meningoencephalitic group and healthy group; $\mathrm{N} / \mathrm{H}$, Differential proteins between the non-meningoencephalitic group and healthy group.

(44.3\%), lysosome (38.8\%), mitochondrion (30.8\%), cytoplasm $(65.5 \%)$, centrosome $(17.5 \%)$, cytosol $(23.4 \%)$, cytoskeleton (8.9\%), nucleolus (15.8\%), mitochondrial matrix (3.2\%), and microtubule (3.9\%) (Figure 3A). The $10 \mathrm{GO}$ terms in cellular biological process contained energy pathway (25\%), metabolism (25\%), protein metabolism (12.5\%), transport $(10.7 \%)$, cell growth and/or maintenance $(9.4 \%)$, protein folding (0.6\%), hormone metabolism $(0.3 \%)$, lipid metabolism $(0.8 \%)$, regulation of exocytosis $(0.3 \%)$ and electron transport $(0.3 \%)$ (Figure 3B). The $10 \mathrm{GO}$ terms in molecular function contained 


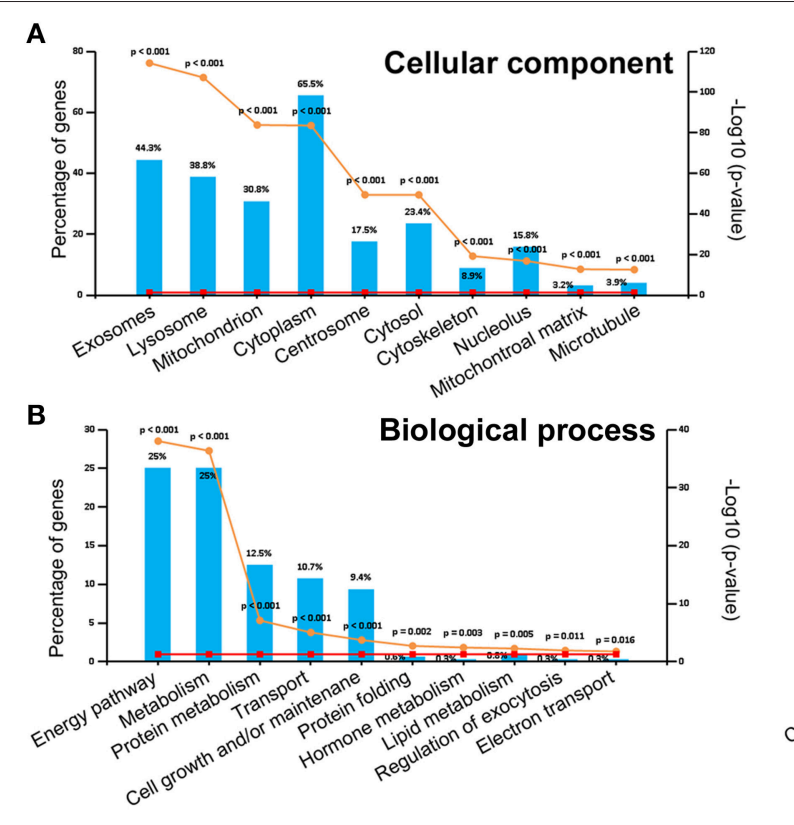

FIGURE 3 | Gene ontology (GO) analysis of 813CDPs. (A) Percentage and $p$-value of proteins in cellular component. (B) Percentage and $p$-value of proteins in biological process. (C) Percentage and $p$-value of proteins in molecular function.

catalytic activity (11.2\%), GTPase activity (4.8), chaperone activity (3.2\%), ATPase activity (2.7\%), structural constitute of cytoskeleton (2.9\%), transporter activity (6.8\%), ligase activity (2\%), calcium ion binding (2.6\%), and hydrolase activity (2.6\%) (Figure 3C). The 813CDPs contain 52 up-regulated proteins (Table 1, Supplementary Material Data Sheet 1) and 761 down-regulated proteins (Supplementary Material Data Sheet 2). The most enriched subclasses of upregulated proteins were extracellular (62.1\%), protein metabolism (34.5\%) and cysteine-type peptidase activity (6.9\%), and of downregulated proteins were exosomes (45.0\%), energy pathway (24.0\%), and catalytic activity (9.4\%) (Supplementary Material Data Sheet 1). The 52 up-regulated proteins contain 27 characterized proteins and 25 uncharacterized proteins (Table 1).

\section{Analysis of Highly Enriched Kyoto Encyclopedia of Genes and Genomes (KEGG)}

The $813 \mathrm{CDP}$ sere further investigated by using the KEGG database and were found to participate in 16 KEGGs (Figure 4, Supplementary Material Data Sheet 3). The most enriched KEGG was citrate cycle (56.6\%). Interestingly, 49 of $813 \mathrm{CDPs}$ are associated with neurological diseases such as Huntington's disease (18.6\%), Parkinson's disease (23.8\%) and Alzheimer's disease (17.6\%). And 23 of 49 differential proteins (genes name: ATP5A1, ATP5B, ATP5F1, COX5B, COX6B, CYCS, LOC100524613, LOC100525869, LOC733678, NDUFA10, NDUFA12, NDUFA8, NDUFA9, NDUFB10, NDUFB4, NDUFC2, NDUFS1, NDUFS2, NDUFS3, NDUFS7, NDUFV1, $\mathrm{SDHA}, \mathrm{SDHB}$ ) are co-exsit in KEGGs of the three neurological diseases (Figure 5). The other enriched KEGGs were Oxidative phosphorylation (25.8\%), Collecting duct acid secretion (30.7\%), Synaptic vesicle cycle (44.05\%), Phagosome (14.8\%), Central carbon metabolism in cancer $(21 \%)$, Pyruvate metabolism (27\%), Glycosis/Gluconeogenesis (29.2\%), Gap junction (17.8\%), Long-term depression (20.3\%), Aminoacyl-tRNA biosynthesis (19.1\%), Valine, leucine and isoleucine degradation $(22.4 \%)$ and Endocrine and other-factor regulated calcium reabsorption (33.3\%) (Figure 4).

\section{Verification of a Subset of Differential Proteins by ELISA}

According to the previous reports, some differential proteins were selected to be identified by ELISA $(n=6)$. The detection results for 7 up-regulated proteins (CTSL1, CTSZ, SAA, MMP2, RBP-4, CHGA, CuZnSOD) and 9 down-regulated proteins (Rock, Clathrin, Vinculin, Arp2/3, RhoA, Bid, CytC, PKC, ERK) are in accord with the results of proteomic analysis (Figure 6).

\section{DISCUSSION}

The SS2 meningoencephalitic pig model was successfully established in this study, and differential protein profiling of CSF in meningoencephalitic, non-meningoencephalitic and healthy pigs was analyzed by label-free proteomic analysis technology. A total of 813 co-differential proteins were found when analyzing the meningoencephalitic group with the nonmeningitic group against the meningoencephalitic group with the healthy group. Seven up-regulated proteins and 9 downregulated proteins of these 813 co-differential proteins were further confirmed by ELISA to support the proteomic data. The 
TABLE 1 | Proteins with special higher LFQ intensity value in meningoencephalitis group.

\begin{tabular}{|c|c|c|c|c|c|}
\hline Uniprot ID & iBAQ intensity & Fold $(M / N)$ & Fold (M/H) & Protein name & Gene name \\
\hline P02189 & 1173900000 & 14.72 & 2.22 & Myoglobin & MB \\
\hline P20112 & 89256000 & 2.19 & 2.73 & SPARC & SPARC \\
\hline P79263 & 6054900000 & 3.87 & 5.09 & Inter-alpha-trypsin inhibitor heavy chain $\mathrm{H} 4$ & ITIH4 \\
\hline Q28944 & 130630000 & 2.50 & 7.64 & Cathepsin L1 & CTSL1 \\
\hline Q7M329 & 75512000 & 2.19 & 11.90 & Ribonuclease T2 & RNASET2 \\
\hline I3LRJ4 & 56294000 & 2.11 & 3.30 & Vitamin K-dependent protein C & PROC \\
\hline F1SD66 & 40023000 & 3.09 & 5.46 & Chromogranin-A & CHGA \\
\hline F1SH92 & 1744300000 & 4.84 & 5.78 & Inter-alpha-trypsin inhibitor heavy chain $\mathrm{H} 4$ & $\mathrm{ITIH} 4$ \\
\hline AOAOA7BZH3 & 59116000 & 4.46 & 4.66 & MHC class I antigen & SLA-1 \\
\hline A5GFX7 & 20490000 & 9.61 & 9.78 & Cathepsin Z & CTSZ \\
\hline A6N9J9 & 172710000 & 3.73 & 6.59 & Secreted phosphoprotein 1 & SPP1 \\
\hline A7YX24 & 776620000 & 2.65 & 3.70 & Gamma-synuclein & SNCG \\
\hline A8D737 & 65674000 & 2.40 & 5.26 & T-cadherin & $N / A$ \\
\hline B5KJG2 & 55810000 & 8.35 & 3.37 & Phosphoglycerate mutase & PGAM2 \\
\hline F1RI57 & 203610000 & 2.82 & 4.82 & Beta-1,3-N-acetylglucosaminyltransferase & LFNG \\
\hline K7GQL2 & 11579000 & 4.20 & 90.59 & Coagulation factor XIII, A1 polypeptide & LOC100153504 \\
\hline Q007T6 & 57101000 & 3.60 & 11.76 & Superoxide dismutase [Cu-Zn] & $N / A$ \\
\hline Q28936 & 329650000 & 3.81 & 6.62 & Fibrinogen A-alpha-chain & N/A \\
\hline Q2HXZ9 & 112470000 & 10.30 & 18.27 & Serum amyloid A protein & LOC733603 \\
\hline T2HRE7 & 47316000 & 4.71 & 3.83 & MHC class I antigen & SLA-2 \\
\hline F1RTPO & 34001000 & 2.87 & 42.97 & Uncharacterized protein & $\mathrm{N} / \mathrm{A}$ \\
\hline F1RTR7 & 58848000 & 2.84 & 3.41 & Uncharacterized protein & YOD1 \\
\hline F1RUQ0 & 726580000 & 2.22 & 10.99 & Uncharacterized protein & JCHAIN \\
\hline F1RVH7 & 111420000 & 2.13 & 3.08 & Uncharacterized protein & IGFBP7 \\
\hline F1RW32 & 22821000 & 2.67 & 5.53 & Uncharacterized protein & SPARCL1 \\
\hline F1S3H9 & 8261500 & 27.27 & 8.86 & Uncharacterized protein & LOC100517145 \\
\hline F1S604 & 29484000 & 6.49 & 3.12 & Uncharacterized protein & $N / A$ \\
\hline F1S8V7 & 21334000 & 2.77 & 6.84 & Uncharacterized protein & CPN1 \\
\hline F1SCC6 & 5888200000 & 2.67 & 3.04 & Uncharacterized protein & LOC100153899 \\
\hline $\mathrm{F} 1 \mathrm{SCC} 7$ & 8583000000 & 2.02 & 2.65 & Uncharacterized protein & LOC100156325 \\
\hline F1SI48 & 74075000 & 2.85 & 6.00 & Uncharacterized protein & EPB42 \\
\hline F1SJY2 & 85340000 & 91.08 & 38.89 & Uncharacterized protein & TMX2 \\
\hline F1SQX9 & 856190000 & 4.918 & 4.44 & Uncharacterized protein & APOD \\
\hline F1SUU7 & 54204000 & 2.06 & 3.97 & Uncharacterized protein & DDI2 \\
\hline I3LBZ1 & 268100000 & 2.29 & 3.51 & Uncharacterized protein & $N / A$ \\
\hline I3LEB3 & 788240000 & 2.25 & 4.75 & Uncharacterized protein & PCSK1N \\
\hline I3LGD9 & 11528000 & 6.26 & 6.21 & Uncharacterized protein & AGRN \\
\hline I3LNM9 & 83333000 & 2.87 & 38.28 & Uncharacterized protein & LOC100624077 \\
\hline
\end{tabular}

Fold (M/N) means LFQ intensity fold change of protein abundance between the meningoencephalitic group and non-meningoencephalitic group; Fold (M/H) means $L F Q$ intensity fold change of protein abundance between the meningoencephalitic group and healthy group. 


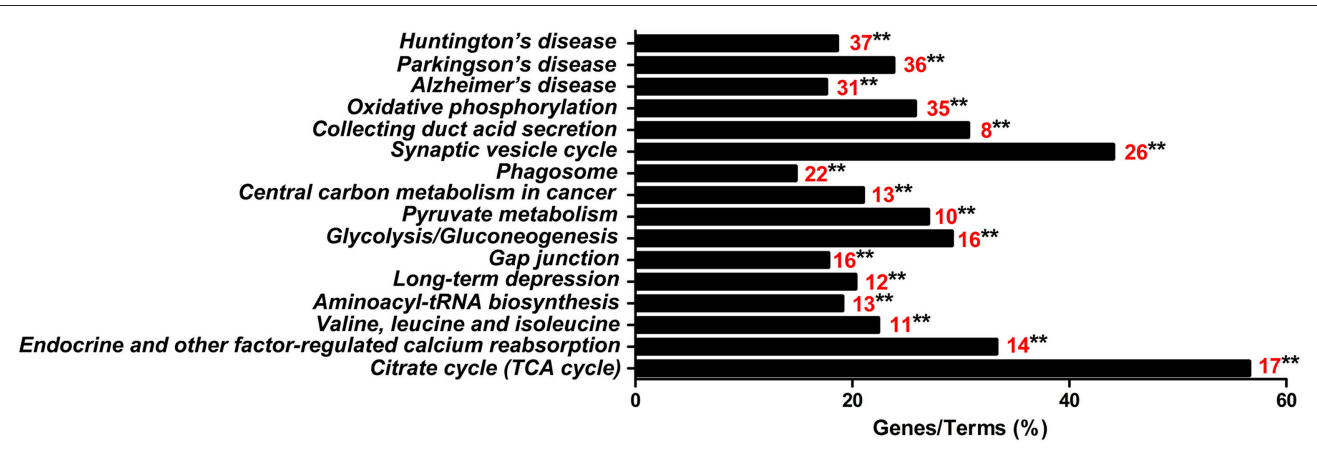

FIGURE 4 | KEGG analysis of the 813 differential proteins. Red number indicates the number of proteins associated with each pathway $\left(^{* \star} p<0.01\right)$.

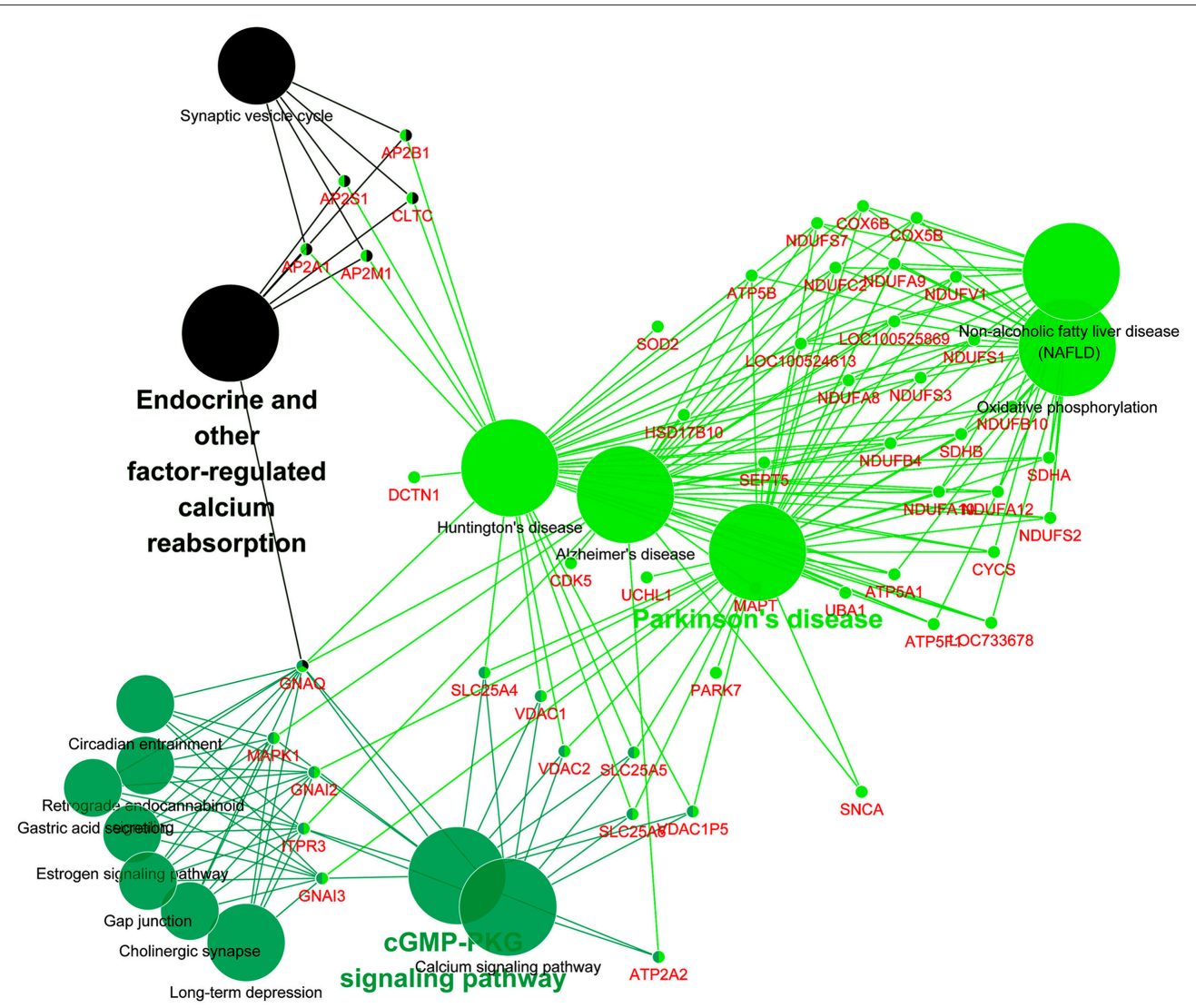

FIGURE 5 | KEGG analysis of differential proteins involved in neurological diseases. Each small dot represents a gene, and each large dot represents a pathway.

data provided important hints for research associated with SS2 meningoencephalitis.

The JZLQ022 strain was isolated from brain tissue of meningoencephalitis pig, and the JZLQ001 strain was from lymphonodi mandibulares of arthritis pig. In our preexperiments, we found that JZLQ022 mainly cause meningitis and JZLQ001 mainly causes arthritis in pigs. Therefore, two SS2 strains, JZLQ022 and JZLQ001, were used to build the meningoencephalitic pig model and non-meningoencephalitic pig model respectively. The model used in this study was a hemorrhagic meningoencephalitis based on results of autopsy (Figure 1A), JZLQ022 strain not only induced meningitis, but also induced severe meningoencephalitis and hemorrhagic encephalitis by crossing BBB and/or BCB. So the differences between the two strains should be analyzed in the future which would provide important implications on mechanism of SS2 across BBB and/or BCB.

Seven up-regulated proteins (CTSL1, CTSZ, SAA, MMP-2, RBP-4, CHGA, and CuZnSOD) in the CSF of meningoencephalitic piglets compared with both 

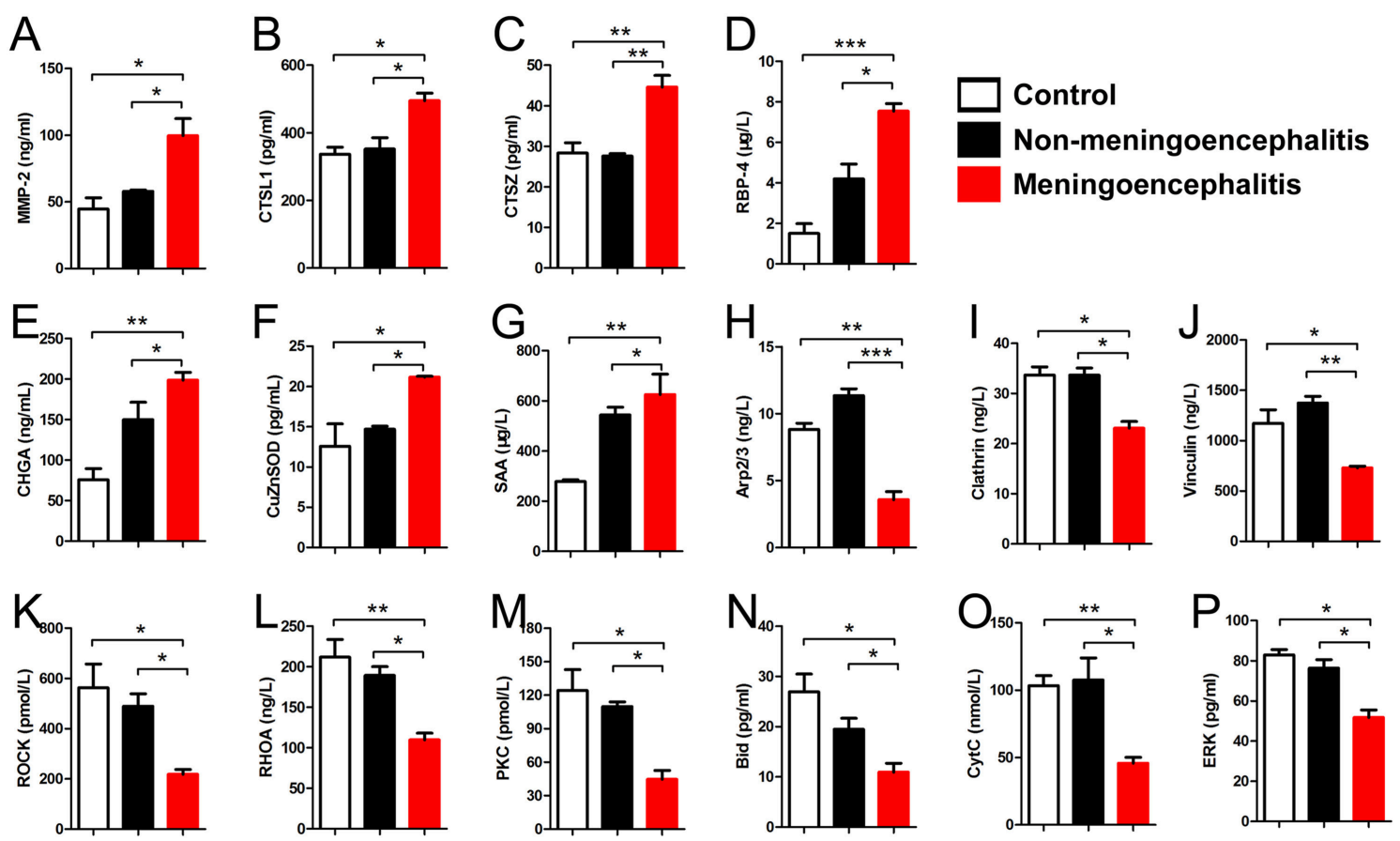

FIGURE 6 | Detection results of differential proteins in the meningitis group by ELISA. (A-G) Special up-regulated proteins in meningoencephalitis group. (H-P) Special down-regulated proteins in meningoencephalitis group. ${ }^{\star} p<0.05 ;{ }^{* \star} p<0.01 ;{ }^{\star \star *} p<0.001$.

non-meningoencephalitic and healthy piglets were found and confirmed by ELISA. One of these proteins, matrix metalloproteinase-2 (MMP-2), is also called $72 \mathrm{kDa}$ type IV collagenase or gelatinase A (Devarajan et al., 1992). There are no previous reports about the relationship between levels of CSF MMP-2 and bacterial meningitis. However, previous reports show that increased levels of serum MMP-2 may reflect the degree of damage to the $\mathrm{BCB}$ in bacterial meningitis (Kanoh et al., 2008). Analysis of MMP levels in the CSF indicates early tumor recurrence and can be used to diagnose CNS tumors, such as malignant astrocytomas, brain metastases, and carcinomatous meningitis (Friedberg et al., 1998). Cathepsin Z (CTSZ) is a member of the cysteine cathepsin protease family (Turk et al., 2012) and cathepsin L1 (CTSL1) is a lysosomal cysteine protease that plays a major role in intracellular protein catabolism. There are no reports on the role of CTSZ and CTSL1 in SS meningitis. However, cathepsin B in CSF from patients has been reported to be associated with neurologic diseases (Nagai et al., 2000). Superoxide dismutase [Cu-Zn], also known as superoxide dismutase 1 or SOD1, is one of three superoxide dismutases in humans. It is implicated in apoptosis and amyotrophic lateral sclerosis (Rosen, 1993). Superoxide dismutase [Cu-Zn] levels were markedly elevated in various neurological diseases, such as bacterial meningitis and encephalitis, and their varied magnitudes may be associated with the underlying diseases (Yoshida et al., 1994). Retinol binding protein 4 (RBP4) is a protein that is encoded in humans by the RBP4 gene (Rocchi et al., 1989). Susceptibility to diet-induced obesity and glucose intolerance in the APP (SWE)/PSEN1
(A246E) mouse model of Alzheimer's disease is associated with increased RBP4 (Mody et al., 2011). Inflammation-dependent cerebral deposition of serum amyloid, a protein, was found in a mouse model of amyloidosis. Inflammation plays an important role in the process of amyloid deposition, and inhibition of inflammatory cascades may attenuate amyloidogenic processes, such as Alzheimer's disease (Guo et al., 2002). Chromogranin A, also called parathyroid secretory protein 1 , is a member of the granin family of neuroendocrine secretory proteins, which is encoded by the CHGA gene (Helman et al., 1988). Elevated levels of Chromogranin A in CSF from patients were associated with Astrocytes of Multiple Sclerosis White Matter Lesions (van Luijn et al., 2016).

Nine down-regulated proteins (Rock, Clathrin, Vinculin, Arp2/3, RhoA, Bid, CytC, PKC, ERK) in the CSF of meningitic piglets compared with both non-meningitic and healthy piglets were found and further confirmed by ELISA. Rho/Rock and PKC signaling pathway have been reported to mediated pathogen breaking through the BBB. For example, globotriaosylceramide (Gb3), which interacts with SS2 Fhb to mediate SS2 penetration into the BBB, needs the activation of the Rho/ROCK signaling pathway (Kong et al., 2017). PKC mediates the penetration of Escherichia coli $\mathrm{K} 1$ and some fungi species in bacterial meningitis (Sukumaran and Prasadarao, 2002; Kim et al., 2012; Salmeri et al., 2012). Vinculin is associated with focal adhesion and adherens junctions (Xu et al., 1998) which are important for maintenance of $\mathrm{BBB}$ function. The loss of vinculin impacts a variety of cell functions; it can disrupt the formation of the complex, and prevents cell adhesion and spreading. The 
absence of vinculin demonstrates a decrease in cell spreading (Goldmann and Ingber, 2002). However, there are no reports about the relationship between clathrin/vinculin and meningitis. Cytoskeleton rearrangement is important for the maintenance of $\mathrm{BCB}$ and BBB function. The Arp2/3 complex is a sevensubunit protein complex that plays a major role in the regulation of the actin cytoskeleton and is found in most actin cytoskeleton-containing eukaryotic cells (Veltman and Insall, 2010). Arp $2 / 3$ complex-regulated actin rearrangement mediates methamphetamine-induced occludin endocytosis (Park et al., 2013). Previous reports indicate that apoptosis is involved in bacterial meningitis (Xu et al., 2017a,b). In this study, Bid and CytC were down-regulated in the CSF of meningitic piglets compared with both non-meningitic and healthy piglets. $\mathrm{Bid}$, the $\mathrm{BH} 3$ interacting-domain death agonist, is a proapoptotic member of the Bcl-2 protein family (Wang et al., 1996). Cytochrome c (cytC) is an electron-transfer protein that possesses a wide range of properties and functions in a large number of different redox processes (Moore et al., 1986). Bid and CytC are important regulators of apoptosis (Chipuk et al., 2012). This suggests that apoptosis may play a role in the process of SS2 breakthrough $\mathrm{BCB}$ or $\mathrm{BBB}$.

Furthermore, 23 differential proteins (genes name: ATP5A1, ATP5B, ATP5F1, COX5B, COX6B, CYCS, LOC100524613, LOC100525869, LOC733678, NDUFA10, NDUFA12, NDUFA8, NDUFA9, NDUFB10, NDUFB4, NDUFC2, NDUFS1, NDUFS2, NDUFS3, NDUFS7, NDUFV1, SDHA, SDHB) which are involved in brain diseases such as Huntington's disease, Parkinson's disease, and Alzheimer's disease (Figure 5) were selected. This suggests that there may be a common mechanism between these brain diseases and meningitis. But the role of

\section{REFERENCES}

Asara, J. M., Christofk, H. R., Freimark, L. M., and Cantley, L. C. (2008). A label-free quantification method by MS/MS TIC compared to SILAC and spectral counting in a proteomics screen. Proteomics 8, 994-999. doi: 10.1002/pmic.200700426

Bantscheff, M., Schirle, M., Sweetman, G., Rick, J., and Kuster, B. (2007). Quantitative mass spectrometry in proteomics: a critical review. Anal. Bioanal. Chem. 389, 1017-1031. doi: 10.1007/s00216-007-1486-6

Boeckmann, B., Bairoch, A., Apweiler, R., Blatter, M. C., Estreicher, A., Gasteiger, E., et al. (2003). The SWISS-PROT protein knowledgebase and its supplement TrEMBL in 2003. Nucleic Acids Res. 31, 365-370. doi: 10.1093/nar/ gkg095

Bradford, M. M. (1976). A rapid and sensitive method for the quantitation of microgram quantities of protein utilizing the principle of proteindye binding. Anal. Biochem. 72, 248-254. doi: 10.1016/0003-2697(76) 90527-3

Brochner, C. B., Holst, C. B., and Mollgard, K. (2015). Outer brain barriers in rat and human development. Front. Neurosci. 9:75. doi: 10.3389/fnins.2015.00075

Chipuk, J. E., McStay, G. P., Bharti, A., Kuwana, T., Clarke, C. J., Siskind, L. J., et al. (2012). Sphingolipid metabolism cooperates with BAK and BAX to promote the mitochondrial pathway of apoptosis. Cell 148, 988-1000. doi: 10.1016/j.cell.2012.01.038

Coureuil, M., Lecuyer, H., Bourdoulous, S., and Nassif, X. (2017). A journey into the brain: insight into how bacterial pathogens cross blood-brain barriers. Nat. Rev. Microbiol. 15, 149-159. doi: 10.1038/nrmicro.2016.178 these proteins in process of SS2 meningitis need to be further investigated.

The present work is the first study, to our knowledge, to analyze the differential protein profiling of CSF in SS2 meningoencephalitic pigs compared with both nonmeningoencephalitic pigs and healthy pigs. The data provide a theoretical basis for diagnosis, pathogenesis and drug therapy research of SS2 CNS infection. Further studies need to clarify the role of these differential proteins in the process of SS2 meningoencephalitis, though some of these proteins have been reported to be associated with neurological diseases.

\section{AUTHOR CONTRIBUTIONS}

Designed the experiments: LL. Performed the experiments: HL, LJ, YS, RZ, WG, SL, and GQ. Conducted the analysis: HL, LJ, HJ, and JW. Provided the analysis tools and technical support: JG, CS, XF, and WH. Wrote and revised the manuscript: HL and LJ. All authors read and approved the final manuscript.

\section{FUNDING}

This study was supported by the National Key R\&D Program of China (2017YFD0500204).

\section{SUPPLEMENTARY MATERIAL}

The Supplementary Material for this article can be found online at: https://www.frontiersin.org/articles/10.3389/fcimb. 2018.00035/full\#supplementary-material

Craft, S., Peskind, E., Schwartz, M. W., Schellenberg, G. D., Raskind, M., and Porte, D. J. (1998). Cerebrospinal fluid and plasma insulin levels in Alzheimer's disease: relationship to severity of dementia and apolipoprotein E genotype. Neurology 50, 164-168.doi: 10.1212/WNL.50.1.164

de Buhr, N., Reuner, F., Neumann, A., Stump-Guthier, C., Tenenbaum, T., Schroten, H., et al. (2017). Neutrophil extracellular trap formation in the Streptococcus suis-infected cerebrospinal fluid compartment. Cell. Microbiol. 19:e12649. doi: 10.1111/cmi.12649

Dejace, J., Bagley, P., and Wood, E. (2017). Streptococcus suis meningitis can require a prolonged treatment course. Int. J. Infect. Dis. 65, 34-36. doi: 10.1016/j.ijid.2017.09.011

Devarajan, P., Johnston, J. J., Ginsberg, S. S., Van Wart, H. E., and Berliner, N. (1992). Structure and expression of neutrophil gelatinase cDNA. Identity with type IV collagenase from HT1080 cells. J. Biol. Chem. 267, 25228-25232.

Friedberg, M. H., Glantz, M. J., Klempner, M. S., Cole, B. F., and Perides, G. (1998). Specific matrix metalloproteinase profiles in the cerebrospinal fluid correlated with the presence of malignant astrocytomas, brain metastases, and carcinomatous meningitis. Cancer-Am. Cancer Soc. 82, 923-930. doi: 10.1002/(SICI)1097-0142(19980301)82:5<923::AID-CNCR18>3.0.CO;2-2

Gahoi, N., Malhotra, D., Moiyadi, A., Varma, S. G., Gandhi, M. N., and Srivastava, S. (2017). Multi-pronged proteomic analysis to study the glioma pathobiology using cerebrospinal fluid samples. Proteomics Clin. Appl. 1700056. doi: 10.1002/prca.201700056

Goldmann, W. H., and Ingber, D. E. (2002). Intact vinculin protein is required for control of cell shape, cell mechanics, and rac-dependent 
lamellipodia formation. Biochem. Biophys. Res. Commun. 290, 749-755. doi: 10.1006/bbrc.2001.6243

Guo, J. T., Yu, J., Grass, D., de Beer, F. C., and Kindy, M. S. (2002). Inflammationdependent cerebral deposition of serum amyloid a protein in a mouse model of amyloidosis. J. Neurosci. 22, 5900-5909.

Helman, L. J., Ahn, T. G., Levine, M. A., Allison, A., Cohen, P. S., Cooper, M. J., et al. (1988). Molecular cloning and primary structure of human chromogranin A (secretory protein I) cDNA. J. Biol. Chem. 263, 11559-11563.

Kanoh, Y., Ohara, T., Kanoh, M., and Akahoshi, T. (2008). Serum matrix metalloproteinase-2 levels indicate blood-CSF barrier damage in patients with infectious meningitis. Inflammation 31, 99-104. doi: 10.1007/s10753-007-9054-y

Kim, J. C., Crary, B., Chang, Y. C., Kwon-Chung, K. J., and Kim, K. J. (2012). Cryptococcus neoformans activates RhoGTPase proteins followed by protein kinase $\mathrm{C}$, focal adhesion kinase, and ezrin to promote traversal across the bloodbrain barrier. J. Biol. Chem. 287, 36147-36157. doi: 10.1074/jbc.M112.389676

Kong, D., Chen, Z., Wang, J., Lv, Q., Jiang, H., Zheng, Y., et al. (2017). Interaction of factor H-binding protein of Streptococcus suis with globotriaosylceramide promotes the development of meningitis. Virulence, 8, 1290-1302. doi: 10.1080/21505594.2017.1317426

Madsen, L. W., Svensmark, B., Elvestad, K., Aalbaek, B., and Jensen, H. E. (2002). Streptococcus suis serotype 2 infection in pigs: new diagnostic and pathogenetic aspects. J. Comp. Pathol. 126, 57-65. doi: 10.1053/jcpa.2001.0522

Mai, N. T., Hoa, N. T., Nga, T. V., Linh, L. D., Chau, T. T., Sinh, D. X., et al. (2008). Streptococcus suis meningitis in adults in Vietnam. Clin. Infect. Dis. 46, 659-667. doi: $10.1086 / 527385$

Mody, N., Agouni, A., McIlroy, G. D., Platt, B., and Delibegovic, M. (2011). Susceptibility to diet-induced obesity and glucose intolerance in the APP (SWE)/PSEN1 (A246E) mouse model of Alzheimer's disease is associated with increased brain levels of protein tyrosine phosphatase 1B (PTP1B) and retinolbinding protein 4 (RBP4), and basal phosphorylation of S6 ribosomal protein. Diabetologia 54, 2143-2151. doi: 10.1007/s00125-011-2160-2

Moore, G. R., Pettigrew, G. W., and Rogers, N. K. (1986). Factors influencing redox potentials of electron transfer proteins. Proc. Natl. Acad. Sci. U.S.A. 83, 4998-4999. doi: 10.1073/pnas.83.14.4998

Nagai, A., Murakawa, Y., Terashima, M., Shimode, K., Umegae, N., Takeuchi, H., et al. (2000). Cystatin, C., and cathepsin B in CSF from patients with inflammatory neurologic diseases. Neurology 55, 1828-1832. doi: 10.1212/WNL.55.12.1828

O'Donovan, C., Martin, M. J., Gattiker, A., Gasteiger, E., Bairoch, A., and Apweiler, R. (2002). High-quality protein knowledge resource: SWISS-PROT and TrEMBL. Brief. Bioinformatics 3, 275-284. doi: 10.1093/bib/3.3.275

Park, M., Kim, H. J., Lim, B., Wylegala, A., and Toborek, M. (2013). Methamphetamine-induced occludin endocytosis is mediated by the Arp2/3 complex-regulated actin rearrangement. J. Biol. Chem. 288, 33324-33334. doi: 10.1074/jbc.M113.483487

Rocchi, M., Covone, A., Romeo, G., Faraonio, R., and Colantuoni, V. (1989). Regional mapping of RBP4 to 10q23-q24 and RBP1 to 3q21-q22 in man. Somat. Cell Mol. Genet. 15, 185-190. doi: 10.1007/BF01535081

Rosen, D. R. (1993). Mutations in Cu/Zn superoxide dismutase gene are associated with familial amyotrophic lateral sclerosis. Nature 362, 59-62.

Salmeri, M., Motta, C., Mastrojeni, S., Amodeo, A., Anfuso, C. D., Giurdanella, G., et al. (2012). Involvement of PKCalpha-MAPK/ERK-phospholipase A(2) pathway in the Escherichia coli invasion of brain microvascular endothelial cells. Neurosci. Lett. 511, 33-37. doi: 10.1016/j.neulet.2012.01.031
Sena, E. S., Carvalho, D. A. J., Abrunhosa, J., Almeida, E. S. C., and Arshad, Q. (2017). Pig's ear: streptococcus suis Meningitis and its associated inner ear implications. IDCases 10, 55-57. doi: 10.1016/j.idcr.2017.08.015

Strazielle, N., and Ghersi-Egea, J. F. (2000). Choroid plexus in the central nervous system: biology and physiopathology. J. Neuropathol. Exp. Neurol. 59, 561-574. doi: 10.1093/jnen/59.7.561

Sukumaran, S. K., and Prasadarao, N. V. (2002). Regulation of protein kinase C in Escherichia coli K1 invasion of human brain microvascular endothelial cells. J. Biol. Chem. 277, 12253-12262. doi: 10.1074/jbc.M110740200

Turk, V., Stoka, V., Vasiljeva, O., Renko, M., Sun, T., Turk, B., et al. (2012). Cysteine cathepsins: from structure, function and regulation to new frontiers. Biochim. Biophys. Acta 1824, 68-88. doi: 10.1016/j.bbapap.2011.10.002

van Luijn, M. M., van Meurs, M., Stoop, M. P., Verbraak, E., Wierenga-Wolf, A. F., Melief, M. J., et al. (2016). Elevated expression of the cerebrospinal fluid disease markers chromogranin, a., and clusterin in astrocytes of multiple sclerosis white matter lesions. J. Neuropathol. Exp. Neurol. 75, 86-98. doi: 10.1093/jnen/nlv004

van Samkar, A., Brouwer, M. C., Schultsz, C., van der Ende, A., and van de Beek, D. (2015). Streptococcus suis meningitis: a systematic review and meta-analysis. PLoS Negl. Trop. Dis. 9:e0004191. doi: 10.1371/journal.pntd.0004191

Veltman, D. M., and Insall, R. H. (2010). WASP family proteins: their evolution and its physiological implications. Mol. Biol. Cell 21, 2880-2893. doi: 10.1091/mbc.E10-04-0372

Wang, K., Yin, X. M., Chao, D. T., Milliman, C. L., and Korsmeyer, S. J. (1996). BID: a novel BH3 domain-only death agonist. Genes Dev. 10, 2859-2869. doi: 10.1101/gad.10.22.2859

Wu, C. H., Yeh, L. S., Huang, H., Arminski, L., Castro-Alvear, J., Chen, Y., et al. (2003). The protein information resource. Nucleic Acids Res. 31, 345-347. doi: 10.1093/nar/gkg040

Xu, D., Lian, D., Wu, J., Liu, Y., Zhu, M., Sun, J., et al. (2017a). Brainderived neurotrophic factor reduces inflammation and hippocampal apoptosis in experimental Streptococcus pneumoniae meningitis. J. Neuroinflammation 14:156. doi: 10.1186/s12974-017-0930-6

Xu, D., Lian, D., Zhang, Z., Liu, Y., Sun, J., and Li, L. (2017b). Brainderived neurotrophic factor is regulated via MyD88/NF-kappaB signaling in experimental Streptococcus pneumoniae meningitis. Sci. Rep. 7:3545. doi: $10.1038 / \mathrm{s} 41598-017-03861-z$

Xu, W., Baribault, H., and Adamson, E. D. (1998). Vinculin knockout results in heart and brain defects during embryonic development. Development 125 , 327-337.

Yoshida, E., Mokuno, K., Aoki, S., Takahashi, A., Riku, S., Murayama, T., et al. (1994). Cerebrospinal fluid levels of superoxide dismutases in neurological diseases detected by sensitive enzyme immunoassays. J. Neurol. Sci. 124, 25-31. doi: 10.1016/0022-510X(94)90006-X

Conflict of Interest Statement: The authors declare that the research was conducted in the absence of any commercial or financial relationships that could be construed as a potential conflict of interest.

Copyright ( 2018 Liu, Jia, Guo, Sun, Zhu, Li, Qu, Jiang, Wang, Gu, Sun, Feng, Han and Lei. This is an open-access article distributed under the terms of the Creative Commons Attribution License (CC BY). The use, distribution or reproduction in other forums is permitted, provided the original author(s) and the copyright owner are credited and that the original publication in this journal is cited, in accordance with accepted academic practice. No use, distribution or reproduction is permitted which does not comply with these terms. 